Trauma Berufskrankh 2006 · 8[Suppl 2]: S207-S211 DOI 10.1007/s10039-005-1023-9

Online publiziert: 2. Juli 2005

(c) Springer Medizin Verlag 2005

\section{K.-H. Thielke · V. Echtermeyer}

Unfallchirurgische Klinik, Kliniken im Mühlenkreis, Klinikum Minden

\title{
Konservatives Vorgehen bei körperfernem Speichenbruch
}

\section{Indikationen im Wandel der therapeutischen Strategien}

\section{Hintergrund und Fragestellung}

Die Entscheidung zum konservativen bzw. operativen Vorgehen in der Versorgung des körperfernen Speichenbruchs ist in erster Linie abhängig vom

- Frakturtyp,

- evtl. bestehenden Begleitverletzungen,

- der Knochenqualität sowie

- vorwiegend vom manuellen Anforderungsprofil des Patienten.

\section{Klassifikation}

Zur Befundbesprechung, Festlegung der Therapie und zum Vergleich der Resultate ist eine gemeinsame Einteilung, d. h. eine einheitliche Sprache, Grundvoraussetzung.

Hier hat sich die AO-Klassifikation [14] immer mehr durchgesetzt. Die Einteilung in Frakturklassen erfolgt anhand der Unfallaufnahmen. Zur Auswertung kommen

- die einfachen extraartikulären Frakturen (Typ A),

- die partiell artikulären Frakturen (Typ B) sowie

- die komplexen artikulären Frakturen (Typ C).

Entsprechend der hierarchischen Gliederung der AO-Klassifikation folgen Grup- pen und Untergruppen. Bei den Typ-AFrakturen lassen sich zunächst die A1Verletzungen, d. h. die isolierten distalen Ulnafrakturen aus dieser Studie ausschließen. Die A2-Fraktur ist die einfache extraartikuläre Radiusfraktur mit dorsaler (Typ A2.2, Colles-Fraktur [4]) bzw. palmarer (Typ A2.3, Smith-Fraktur [17]) Abkippung des distalen Fragments. Die extraartikulären Frakturen der Speiche mit metaphysärer Trümmerzone sind unter A3 angeführt.

Bei den Typ B-Verletzungen erfolgt die Unterteilung nach Richtung der Frakturebene. Bei der Gruppe B1 verläuft sie in sagittaler Richtung (Chauffeur-Fraktur). In der Frontalebene zeigt die B2-Verletzung eine Fraktur der dorsalen (BartonFraktur [1]), die B3-Verletzung eine Fraktur der palmaren Kante (reversed BartonFraktur).

Die Typ C-Verletzungen sind die typischen Gelenkfrakturen. Bei den C1-Frakturen sind die Gelenkfläche und die Metaphyse einfach frakturiert. In der Gruppe C2 findet sich eine Mehrfachfraktur der Metaphyse mit einer Einfachfraktur der Gelenkfläche. Unter C3 werden alle Frakturen mit mehrfach frakturierter Gelenkfläche zusammengefasst.

\section{Therapie}

Ihre Ziele beim körperfernen Speichenbruch sind
- die exakte Fragmentreposition,

- eine ausreichende Stabilität (Retention) und

- eine rasche Wiederherstellung der Funktion (Rehabilitation, frühfunktionelle Nachbehandlung).

Die Indikation zum rein konservativen Vorgehen ist damit begrenzt. Entsprechend der Einteilung der distalen Radiusfrakturen nach der AO-Klassifikation werden in der Unfallchirurgischen Klinik am Klinikum Minden nur die stabilen A2Frakturen konservativ behandelt. In Ausnahmefällen werden extraartikuläre Frakturen ohne metaphysäre Trümmerzone (A2), die einer Reposition bedürfen, sowie alle $\mathrm{A}_{3}$-Verletzungen mit Kirschner-Drähten in der Technik nach Kapandji [10] gestiftet, und zwar in Kenntnis, dass die geschlossene Reposition meist ohne Probleme gelingt, jedoch die Beibehaltung des Repositionsergebnisses, also die Retention, das eigentliche Problem bei der Behandlung des körperfernen Speichenbruchs darstellt. Lediglich die Smith-Frakturen (A2.3) werden in der Gruppe der extraartikulären Frakturen mit einer palmaren Plattenosteosynthese versorgt.

Die Typ-B-Verletzungen sind mittels Schrauben- (B1) bzw. Plattenosteosynthese $\left(\mathrm{B}_{2}\right.$ und $\left.\mathrm{B}_{3}\right) \mathrm{zu}$ versorgen.

Bei den C-Verletzungen werden primär winkelstabile palmare Plattenosteosynthesen bzw. Kombinationsverfahren 


\section{Körperferner Speichenbruch}
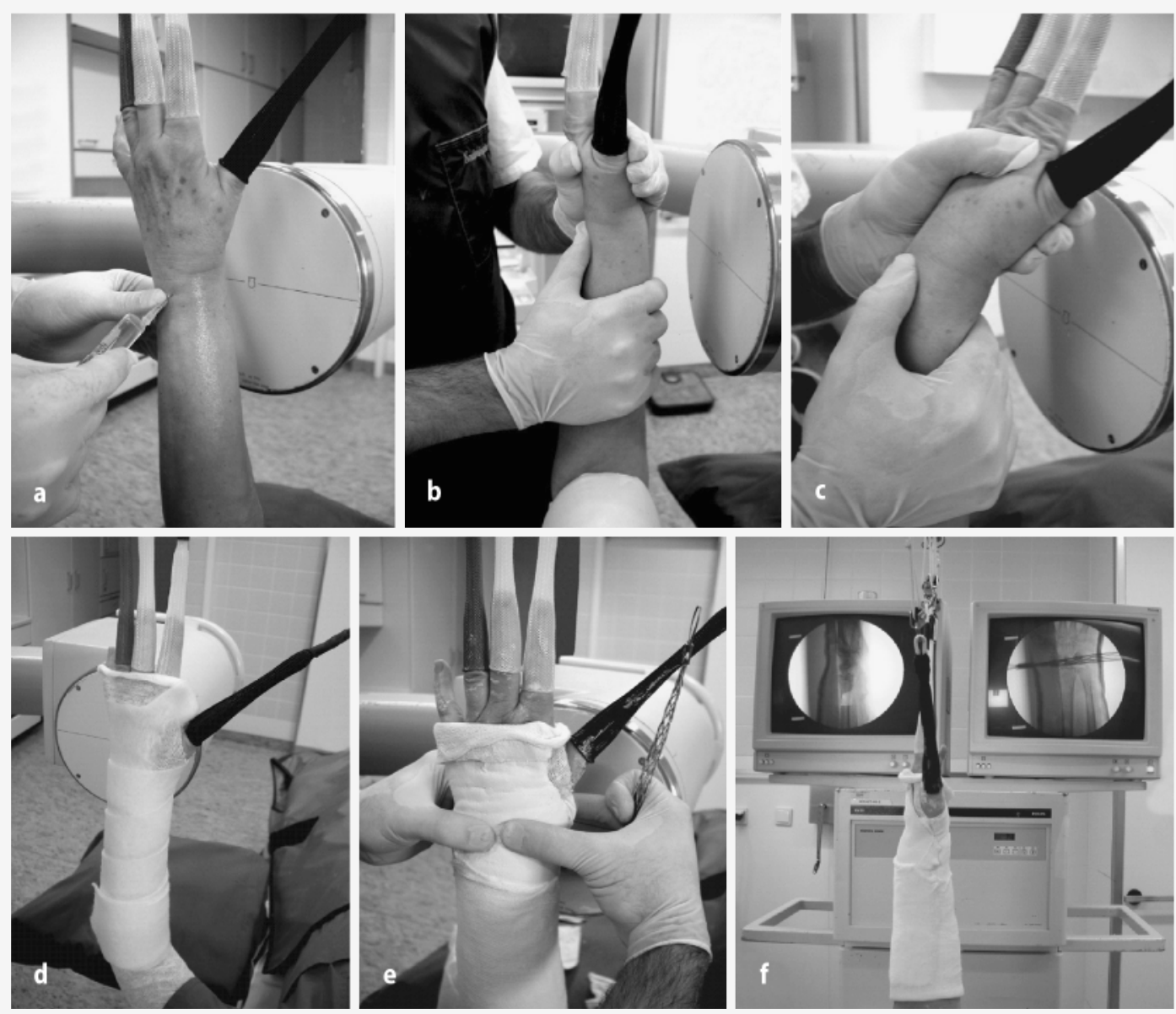

Abb. 1 a $<$ Aushang in „Mädchenfängern", Bruchspaltanästhesie, b,c Reposition nach Charnley, d,e Gipsimmobilisation, 3-Punkt-Abstützung mit Anmodellierung einer dorsaler Delle über der proximalen Handwurzelreihe zur sicheren Retention, fübersichtsbild:Bildwandlerkontrolle des Repositionsergebnisses, sichtbare Delle im Bereich der proximalen Handwurzelreihe dorsal
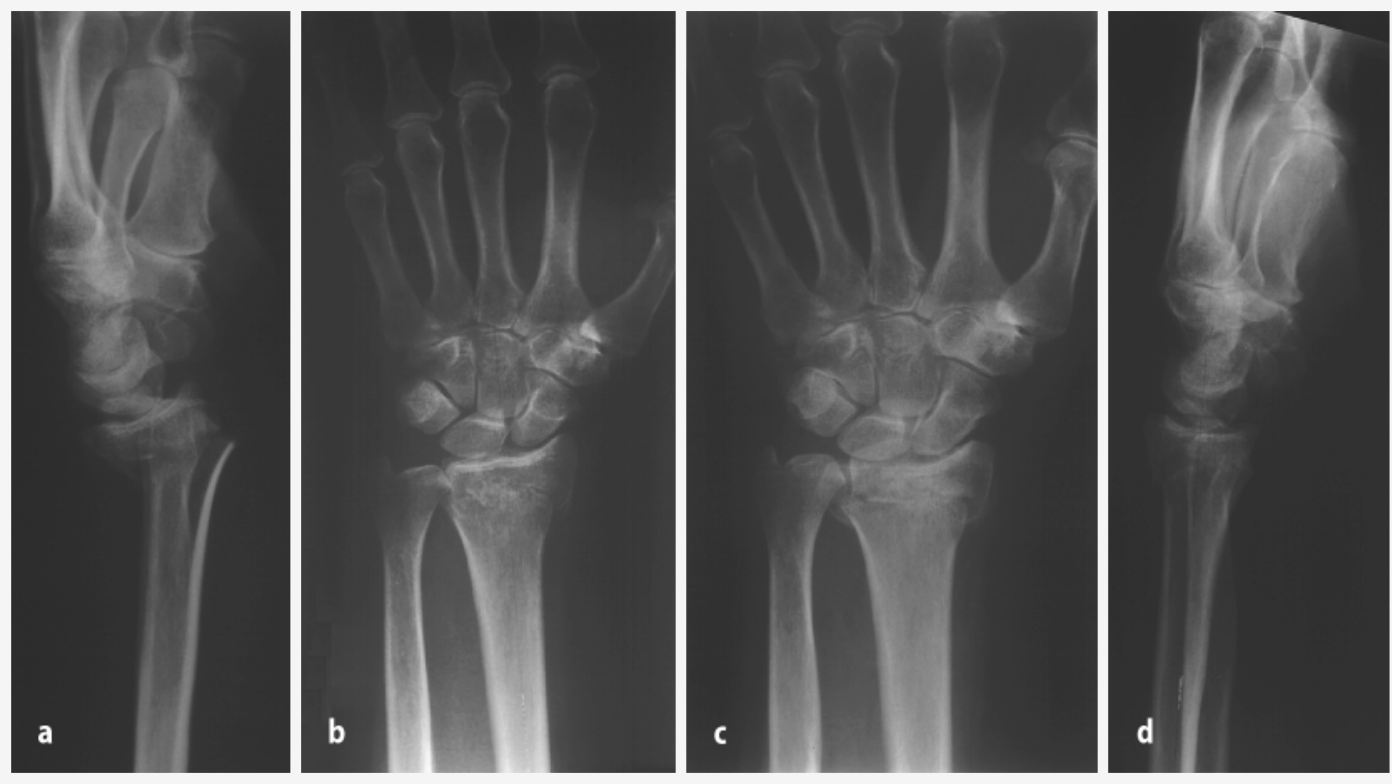

Abb. $2<$ Unfallaufnahmen in 2 Ebenen $(a, b)$ einer 66-jährigen Patientin mit einer A3-Verletzung, nach Ablehnung aller operativen Maßnahmen durch Patientin Durchführung einer rein konservativen Therapie, c,d Zwischenkontrolle bei Anlage eines erneuten zirkulären Baycast am 10. Tag nach dem Unfallereignis mit achsengerechten Stellungsverhältnissen

in Abhängigkeit vom Frakturtyp, eventuellen Begleitverletzungen und dem funktionellen Anspruch des Patienten angewandt.

Das entsprechende Therapieschema der körperfernen Speichenbrüche ist in - Tabelle 1 wiedergegeben.

\section{Konservatives bzw. semioperatives Vorgehen}

Wenn ein körperferner Speichenbruch ambulant konservativ bzw. semioperativ mit zusätzlicher Kirschner-Draht-Stiftung versorgt wird, muss dies nach einem festen Behandlungsablauf und -plan erfolgen.

Voraussetzung für eine optimale Reposition ist eine suffiziente Schmerzausschaltung, ein gängiges und einfaches Verfahren ist die Bruchspaltanästhesie (• Abb. 1b). Wichtig ist dabei, dass die Nadel von proxi- 
mal-dorsal nach distal-palmar vorgeschoben wird, um den Bruchspalt zu erreichen. Nach Punktion des Bruchspalthämatoms werden etwa $10 \mathrm{ml}$ eines 1\%igen Lokalanästhetikums instilliert. Die Lokalanästhesie führt, wie die Schule von Lorenz Böhler anhand von 30.000 Fällen zeigen konnte, zu keinen nennenswerten Komplikationen. Sie bringt eine ausreichende Analgesie, ist einfach durchzuführen und kostengünstig. Das häufig genannte Gegenargument, es werde hierdurch eine geschlossene in eine offene Fraktur umgewandelt, wird durch die positiven klinischen Erfahrungen widerlegt.

Die Reposition erfolgt in Rückenlage. Die eigentliche Einrichtung wird nach dem Prinzip von Zug und Gegenzug unter manuellem Druck ausgeführt. Hilfreich sind die so genannten „Mädchenfänger" (Extensionshülsen), wobei der Zug über den Daumen läuft. Die Extensionshülsen des 2.-4. Fingers dienen als Rotationsschutz des Carpus. Jedoch richtet sich nur ein Teil der Frakturen allein durch Zug und Gegenzug ein. Liegt eine Seitverschiebung vor, muss manuell nachreponiert werden.

Charnley [3] verglich die Frakturflächen des proximalen und distalen Fragments mit den Zähnen zweier Räder, die falsch ineinander greifen. Ist der distale Zahn um 2 Zähne gegen das proximale Rad versetzt, kann einfacher Druck die beiden Räder nicht wieder in die richtige Verzahnung bringen, ohne die Zähne abzuscheren. Vermehrter Zug in Längsrichtung und forcierte Dorsalabwinkelung der Hand (Nachahmen des Unfallmechanismus) führen zur Entzahnung und schaffen damit die Voraussetzung für die Reposition zur Aufhebung der Seitverschiebung (- Abb. 1b,c). Durch diese beiden Bewegungen wird es möglich, die beiden "Zahnräder" in die Ausgangsstellung zurückzubringen. Die so erhaltene bzw. wiederhergestellte Verzahnung ist ein wichtiger Stabilitätsfaktor. Erst danach erfolgt die Winkelkorrektur durch Ulnar- und Palmarduktion. Die Retention wird in einer dorsalen Gipsschiene vorgenommen. Über der Streckseite wird beim Anlegen der Gipsbinde eine Delle in Höhe der proximalen Handwurzelreihe zur Sicherung des Repositionsergebnisses und zur Prophylaxe der Redislokation anmodelliert

Trauma Berufskrankh 2006 · 8[Suppl 2]: S207-S211

DOI 10.1007/s10039-005-1023-9

c) Springer Medizin Verlag 2005

K.-H. Thielke $\cdot$ V. Echtermeyer

Konservatives Vorgehen bei körperfernem Speichenbruch. Indikationen im Wandel der therapeutischen Strategien

\section{Zusammenfassung}

Die distale Radiusfraktur ist mit einer Inzidenz von 2-3/1000 Einwohner/Jahr die häufigste Fraktur des menschlichen Skeletts und damit von hoher sozioökonomischer Relevanz. Das jährliche Frakturaufkommen von etwa 200.000 Fällen führt zu Kosten im weit 3-stelligen Millionen-Euro-Bereich. Als kostengünstiges, ambulantes Verfahren kommt die rein konservative bzw. semioperative Kirschner-Draht-Stiftung in Bruchspaltanästhesie in Frage. Der extraartikuläre, stabile Speichenbruch (Typ A2 nach $A O$ ) sollte konservativ behandelt werden. Die Gefahr der sekundären Dislokation bleibt im Rahmen eines suffizienten Nachbehandlungsschemas minimal. Ist eine Reposition der Fraktur erforderlich, sollte operativ mit zumindest einer perkuta-

\section{Distal radius fractures: indications for conservative therapy. In times of changing therapeutic strategies}

\section{Abstract}

Fractures of the distal radius represent one of the most common fractures, with an incidence of 2-3/1,000/year, and have a high social-economic relevance. There are about 200,000 cases a year with costs reaching the 100 s of millions. For treatment, such fractures need a practical classification and therapeutic standards. For stable A2-fractures, we prefer closed reduction, and only in special cases K-wire fixation. Such fractures are reduced using the technique of Charnley. $A$ plaster cast based on the principle of threepoint fixation is applied to immobilize the nen Kirschner-Draht-Sicherung stabilisiert werden. Diese dann instabilen Extensionsfrakturen am distalen Radius (AO-Klassifikation A3, C1-C3) stellen trotz zahlreicher Behandlungsoptionen nach wie vor eine therapeutische Herausforderung dar. Bei einem klaren Trend zur Operation werden in der Literatur die Indikationen zum jeweiligen Verfahren (auch konservativ) abhängig von Frakturtyp, Begleitverletzungen und manuellem Anforderungsprofil unterschiedlich definiert.

\section{Schlüsselwörter}

Distale Radiusfraktur .

Bruchspaltanästhesie $\cdot$ Konservative

Therapie · Kapandji · Kirschner-Draht

\begin{abstract}
fractured part. Instable and displaced intra-articular fractures according to the $\mathrm{AO}$ scheme type $A 3$ and $C 1-3$ are indications for percutaneous pinning, transfixation or plate osteosynthesis. Differential management has led to a change from purely conservative treatment to a more varied treatment of such fractures.
\end{abstract}

\section{Keywords}

Distal radius fracture $\cdot$ Local anesthesia in the fracture gap · Kapandji .

Conservative treatment $\cdot$ Kirschner wire 
Tabelle 1

Behandlung distaler Radiusfrakturen

\begin{tabular}{|c|c|c|}
\hline \multirow[t]{2}{*}{ Fraktur } & \multicolumn{2}{|l|}{ Behandlung } \\
\hline & Therapie der ersten Wahl & Fakultativ \\
\hline A2 & Konservativ & Kirschner-Draht-Stabilisierung \\
\hline A3 & Kirschner-Draht-Stabilisierung & Plattenosteosynthese \\
\hline B1-3 & Schrauben-/Plattenosteosynthese & Kirschner-Draht-Stabilisierung \\
\hline C1 & Winkelstabile Plattenosteosynthese & Kirschner-Draht-Stabilisierung \\
\hline C2 & Winkelstabile Plattenosteosynthese & Kombinationsverfahren \\
\hline C3 & Kombinationsverfahren & \\
\hline
\end{tabular}

Behandlung an der Unfallchirurgischen Klinik, Klinikum Minden; abhängig von der AO-Klassifikation

(- Abb. 1d-f). Dies ist notwenig, da die geschlossene Reposition meist ohne Probleme gelingt, die Retention aber das eigentliche Problem in der Behandlung der distalen Radiusfrakturen darstellt. Es besteht eine hohe Redislokationsneigung.

Wenn eine Reposition einer extraartikulären Radiusfraktur notwendig ist, sollte auch eine minimalinvasive Osteosynthese (z. B. in Form der Kirschner-Draht-Stabilisierung) durchgeführt werden. Zur Sicherung des Repositionsergebnisses werden Kirschner-Drähte z. B. in der Technik nach Kapandji [10] eingebracht. Bei dieser intrafokalen Spickung werden die Drähte der Stärke 1,8 mm durch den dorsalen Bruchspalt unter manueller Kontrolle vorgeschoben, ggf. distal gekippt, um eine verbesserte Reposition zu erreichen. Erst danach erfolgt die Verankerung in der Gegenkortikalis. Das periphere Fragment wird somit nicht durch die Drähte gefasst, sondern wie durch ein Körbchen in Repositionsstellung gehalten.

Nach Anlage der oben bereits beschriebenen dorsalen Gipsschiene und Röntgenkontrolle verbleibt der Patient in ambulanter Behandlung. Ein zirkulärer Gipsverband wird nach Abschwellung, in der Regel nach 4 Tagen, angelegt und anschließend röntgenologisch kontrolliert. Dabei ist ggf. noch eine Nachreposition möglich. Ein erneuter Gipswechsel sollte nach $10 \mathrm{Ta}-$ gen erfolgen, ebenso eine radiologische Kontrolle in 2 Ebenen.

Der Gips wird nach 5 Wochen abgenommen. 1 Woche später werden die Kirschner-Drähte in Lokalanästhesie entfernt. Krankengymnastische Übungsbehandlungen dienen der Anleitung zu Eigenübungen.
Ein klinisches Beispiel ist in $\bullet \mathbf{A b b} \mathbf{2}$ wiedergeben. Die Patientin erlitt im Rahmen eines typischen Unfallmechanismus einen Sturz auf die dorsal extendierte Hand. Nach ambulanter Abklärung lehnte sie operative Maßnahmen ab, so dass eine rein konservative Therapie mit Reposition unter Bruchspaltanästhesie und sicherer Retention durch Gipsimmobilisation durchgeführt werden musste. Nach unserem Therapieschema hätten wir bei dieser $\mathrm{A}_{3}$-Verletzung eine zusätzliche Sicherung mittels Kirschner-Drähten durchgeführt. Nach entsprechender Ausbehandlung im Gips zeigte sich in der 3nisches, funktionelles und radiologisches Resultat.

\section{Diskussion}

Während Abraham Colles [4] anlässlich seiner Erstbeschreibung der Radiusextensionsfraktur 1814 noch ein gutes Behandlungsergebnis unabhängig vom anatomischen Ausheilungsergebnis prophezeite, zeigt sich im Literaturstudium, dass gerade die betroffenen Gelenkachsen und damit die anatomische Wiederherstellung der regelrechten Artikulation im distalen Radioulnar- und Radiokarpalgelenk die Voraussetzung für ein funktionell gutes $\mathrm{Er}$ gebnis darstellen. Zur Versorgung der distalen Radiusfraktur ist daher ein differenziertes Behandlungsverfahren zu fordern, welches nicht nur den Frakturtyp, sondern auch den funktionellen Anspruch des Unfallverletzten berücksichtigt.

Die rein konservative Behandlung [7] ist insbesondere aufgrund der verbesserten operativen Möglichkeiten nur noch Monats-Kontrolle dennoch ein gutes kli- bei stabilen Frakturen (Typ A2 nach der AO-Klassifikation) zu empfehlen. Hauptgrund ist die hohe Redislokationsneigung der instabilen Frakturen $\left(\mathrm{A}_{3}, \mathrm{C}_{1}-\mathrm{C}_{3}\right)$, auch bei suffizienter Nachbehandlung.

Nach der früher üblichen konservativen Therapie, unabhängig vom Frakturtyp, zeigten sich Anfang der 1990er Jahre in bis zu $30 \%$ der Fälle klinisch und radiologisch schlechte Ergebnisse [2, 6, 8, 11]. Dies führte zu einer Umorientierung in der Behandlung distaler Radiusfrakturen $[5,9,13,18,24]$.

Die Kirschner-Draht-Stabilisierung gewann immer mehr an Bedeutung. Ihre Erstbeschreibung über den Processus styloideus radii wurde von Lambotte [12] veröffentlicht. Der Nachteil dieser konventionellen Stabilisierung bestand allerdings in einer unzureichenden Fixierung des dorsoulnaren Fragments, einem Schlüsselfragment in der Stabilisierung distaler Radiusfrakturen. Die Methode nach Kapandji [10] ermöglicht hingegen eine der Fraktursituation angepasste Platzierung der Kirschner-Drähte und damit ein besseres Outcome $[15,19]$. In unserer Klinik wird dieses Verfahren bevorzugt und evtl. durch vom Processus styloideus radii eingebrachte Drähte insbesondere bei Gelenkbeteiligung ergänzt. Zur Sicherung und Fixierung von Gelenk tragenden Fragmenten werden im Einzelfall auch parallel zur Gelenkfläche eingebrachte Kirschner-Drähte benutzt [20]. Zudem ist diese Form der Stabilisierung ein einfaches, wenig invasives und auch kostengünstiges Verfahren, was gerade in der heutigen Zeit immer mehr an Bedeutung gewinnt.

Die alleinige Versorgung von Typ-CVerletzungen mit Kirschner-Drähten sollte ausgewählten Gelenkfrakturen vorbehalten bleiben, sie ist nicht das Verfahren der 1. Wahl. Neuere operative Möglichkeiten, wie insbesondere die palmare winkelstabile Verplattung [16, 21, 22, 23, 25], sind hier der rein konservativen bzw. semioperativen Behandlung überlegen. Dennoch sind die Kenntnis und Anwendung der konservativen Therapie mit Reposition und fachgerechter Gipsimmobilisation unter Beachtung eines konsequenten Nachbehandlungsregimes in der Ausbildung und Tätigkeit eines Chirurgen zwingend notwendig. 


\section{Fazit für die Praxis}

Eine differenzierte, patientenorientierte Behandlung distaler Radiusfrakturen ist zu fordern. Die Indikation zur konservativen Therapie ist nur bei stabilen, extraartikulären Frakturen (Typ A2 nach A0) zu stellen. Instabile Extensionsfrakturen (A3, C1-C3 nach A0) bedürfen einer Reposition, anatomischen Rekonstruktion und sicheren Retention des Radiokarpalgelenks, um ein gutes Langzeitergebnis zu erzielen. Seitens der extraartikulären Frakturen bieten die semioperativen Kirschner-DrahtStabilisierungen nicht nur eine Alternative, sondern stellen in unserer Klinik das Verfahren der Wahl als ambulanten, kostengünstigen Eingriff dar. Erfordert das manuelle Anforderungsprofil des Patienten eine frühfunktionelle Nachbehandlung, wird man in erster Linie auf winkelstabile palmare Plattenosteosynthesen zurückgreifen. Bei Frakturen mit Gelenkbeteiligungen (Typ-B- und -C-Verletzungen) ist die operative Versorgung als Therapie der 1. Wahl zu betrachten.

\section{Korrespondierender Autor}

\section{Dr. K.-H. Thielke}

Unfallchirurgische Klinik, Kliniken im Mühlenkreis, Klinikum Minden, Friedrichstraße 17, 32427 Minden

E-Mail:Thielke.KH@t-online.de

Interessenkonflikt: Der korrespondierende Autor versichert, dass keine Verbindungen mit einer Firma, deren Produkt in dem Artikel genannt ist, oder einer Firma, die ein Konkurrenzprodukt vertreibt, bestehen.

\section{Literatur}

1. Barton JR (1838) Views and treatment of an important injury of the wrist. Med Examiner Record Med Sci 1838: 367

2. Boszotta H, Helperstorfer W, Sauer G (1991) Zur Operationsindikation bei der distalen Radiusfraktur. Unfallchirurg 94: 417-423

3. Charnley J (1968) Die konservative Therapie der Extremitätenfrakturen. Springer, Berlin Heidelberg New York

4. Colles A (1814) On the fracture of the carpal extremity of the radius. Edinburgh Med Surg J 10: 182186

5. Fitoussi F, Ip WY, Chow SP (1997) Treatment of displaced intra-articular fractures of the distal end of the radius with plates. J Bone Joint Surg Am 79: 1303-1312

6. Gabl M, Pechlaner S, Sailer R et al. (1992) Dorsale Stauchungsbrüche der distalen Radiusmetaphyse Langzeitbeobachtung nach konservativer Therapie. Aktuelle Traumatol 22: 15-18
7. Jakob M, Mielke S, Keller H et al. (1999) Therapieergebnisse nach primär konservativer Versorgung distaler Radiusfrakturen bei Patienten im Alter über 65 Jahren. Handchir Mikrochir Plast Chir 31: 241-245

8. Jansky W, Laminger K, Iqbal M et al. (1994) Klinische und radiologische Ergebnisse von handgelenksnahen Speichenbrüchen. Unfallchirurgie 20 197-202

9. Joosten U, Joist A, Frebel T et al. (1999) Die Behandlung instabiler distaler Radiusfrakturen mit einem transartikulären Fixateur externe: Ergebnisse einer Langzeitbeobachtung. Chirurg 70: 1315-1322

10. Kapandji A (1976) L'osteosynthese par double embrochage intra-focal: traitement fonctionnel des fractures non articulaires de l'extremite inférieure du radius. Ann Chir 30: 903

11. Kwasny O, Barisani R, Schabus R et al. (1991) Ergebnisse und Analyse von Misserfolgen der konservativen Therapie bei distaler Radiusfraktur. Handchir Mikrochir Plast Chir 23: 240-244

12. Lambotte (ed) (1913) Chirurgie opératoire des fractures. Masson, Paris

13. Letsch R, Schmit-Neuerburg KP, Towfigh H (1984) Indikationen und Ergebnisse der Plattenosteosynthese des distalen Radius. Langenbecks Arch Chir 364: 363-368

14. Müller ME, Nazarian S, Koll P (Hrsg) (1987) AO-Klassifikation der Frakturen. Springer, Berlin Heidelberg New York

15. Nonnenmacher J, Neumeier K (1987) Intrafokale Verdrahtung bei Handgelenkfrakturen. Handchir Mikrochir Plast Chir 19: 67-70

16. Sakhaii M, Groenewold U, Klonz A et al. (2003) Ergebnisse der palmaren Plattenosteosynthese mit der winkelstabilen T-Platte bei 100 distalen Radiusfrakturen - Eine prospektive Studie. Unfallchirurg 106: $272-280$

17. Smith RW (ed) (1847) A treatise on fracture in the vicinity of joints and on certain forms of accidental and congenital dislocations. Hodges \& Smith, Dublin, pp 162-163

18. Steffen T, Eugster T, Jakob RP (1994) Twelve years follow-up of fractures of the distal radius treated with the AO external fixator. Injury 25: 44-54

19. Strohm PC, Müller CA, Boll T et al. (2004) Two procedures for Kirschner wire osteosynthesis of distal radius fractures. A randomized trial. J Bone Joint Surg Am 86: 2621-2628

20. Thielke KH, Wagner T, Giers R et al. (2001) Ambulante KD-Stiftung distaler Radiusfrakturen: OutcomeBeurteilung im Vergleich verschiedener ScoringSysteme. Aktuelle Traumatol 31: 256-260

21. Thielke KH, Spors-Schrödter L, Wagner T et al. (2002) Winkelstabile Plattenosteosynthese am distalen Radius: Lösung einer Problemfraktur? Aktuelle Traumatol 32: 1-6

22. Thielke KH, Wagner T, Bartsch S et al. (2003) Winkelstabile, volare Plattenosteosynthese am distalen Radius - Lösung einer Problemfraktur? Chirurg 74: 1057-1063

23. Uzdil T, Neumann W, Bauschke A et al. (2001) Die palmare winkelstabile Plattenosteosynthese bei distalen Radiusextensionsfrakturen. Aktuelle Traumatol 31: 141-148

24. Wiemer P, Köster G, Felderhoff J et al. (1999) Frakturen am distalen Radius. Wandel der therapeutischen Strategien. Orthopäde 28: 846-852

25. Zingg U, Rillmann P, Metzger U et al. (2005) Osteosynthese distaler Radiusfrakturen mit der AO/ASIF Titan-Pi-Platte. Unfallchirurg 108: 206-214 